\title{
Cáncer, neutropenia febril e imágenes pulmonares: Hallazgos en el lavado broncoalveolar en niños
}

\author{
Carmen L. Avilés, Pamela Silva, Marcela Zubieta, Ana M. Álvarez, Ana Becker, Carmen Salgado, \\ M. Elena Santolaya, Santiago Topelberg, Juan Tordecilla, Mónica Varas, Milena Villarroel y Tamara Viviani \\ en representación del Comité de Infectología del PINDA. Ministerio de Salud de Chile
}

Cancer, febrile neutropenia and pulmonary images:

Findings in bronchoalveolar lavage in children

Introduction: Lung infections are a serious complication in children with cancer. Bronchoalveolar lavage (BAL) has been demonstrated to be an effective procedure for achieving etiologic diagnosis. Method: We did a retrospective analysis of BAL data performed between November 2005 and October 2008 in children with cancer, severe neutropenia and lung infiltrates for assessing its performance, clinical utility and safety. Thirty-seven BAL were evaluated in 35 patients. Results: Focal infiltrates were demonstrated in imaging studies associated with 19/37 BAL; in 8 an infectious agent was found. Interstitial pattern was observed in 15/37, in which there were 4 positive studies, proving a higher microbiological performance in BAL associated with focal lesions. BAL yielded significant microbiological findings in 32.4\% (12/37). Sixteen microorganisms were identified in the study: bacteria in 8 cases, Mycobacterium tuberculosis (n: 2), Pseudomonas aeruginosa (n: 2), Acinetobacter baumannii (n: 1), A. lwoffii (n: 1), group viridans Streptococcus (n: 1), Mycoplasma pneumoniae (n: 1); viruses in 3 cases, metapneumovirus (n: 2) cytomegalovirus (n: 1) and fungal infection in 5 cases, Pneumocystis jiroveci (n: 2) Aspergillus fumigatus (n: 1), Aspergillus niger (n: 1), Candida albicans (n: 1). Therapeutic adjustments were done in 6/37 episodes (16.2\%). Conclusion: BAL has a significant role in the evaluation of pulmonary infiltrates in pediatric oncological patients, requiring a prompt and safe diagnosis, which is crucial for the survival with minimal morbidity. Our results suggest that BAL by fiberbronchoscopy should be considered as an initial diagnostic tool in these patients.

Key words: Bronchoalveolar lavage, children, cancer, lung infections, febrile neutropenia.

Palabras clave: Lavado broncoalveolar, niños, cáncer, infecciones pulmonares, neutropenia febril.

\section{Introducción}

$\mathrm{L}$

as infecciones pulmonares en pacientes con cáncer, neutropenia y fiebre constituyen una de las principales causas de mortalidad ${ }^{1}$. El diagnóstico definitivo debe ser efectuado precozmente para obtener evoluciones clínicas favorables. Su estudio etiológico debe considerar causas infecciosas y no infecciosas, encontrándose con frecuencia agentes oportunistas como responsables de esta complicación. El cuadro clínico y los exámenes habitualmente solicitados como hemocultivos, cultivo de secreción bronquial, radiografías y tomografía computarizada de tórax, con frecuencia no permiten un diagnóstico etiológico por lo que se acude a procedimientos como el lavado bronco-alveolar (LBA) para lograr una mayor precisión diagnóstica. Debido a la gravedad potencial de estos pacientes el tratamiento antimicrobiano de amplio espectro es iniciado empíricamente siendo la demostración de la etiología de gran importancia para ajustar o suspender la terapia antimicrobiana ${ }^{2}$.

Se ha demostrado que el LBA por fibro-broncoscopia (FBC) en niños es un procedimiento eficiente que permite confirmar la etiología de cuadros infecciosos pulmonares en forma rápida y confiable, con pocas complicaciones, las que de producirse son usualmente leves y sin mortalidad $^{3-5}$. El procedimiento es efectivo y bien tolerado en pacientes oncológicos incluso en condiciones de insuficiencia respiratoria ${ }^{6-9}$.

El objetivo de este trabajo fue evaluar en forma sistematizada los hallazgos microbiológicos de muestras de LBA, sumando el aporte de métodos diagnósticos por biología molecular, en cuadros de infiltrado pulmonar de niños en tratamiento de quimioterapia por cáncer, conocer su rendimiento, seguridad y utilidad clínica, ya que existe poca información al respecto en nuestro medio.

\section{Pacientes y Métodos}

Se realizó un estudio prospectivo, protocolizado, entre noviembre de 2005 y octubre de 2008 en seis hospitales pediátricos de Santiago, de los LBAs efectuados en pacientes bajo 18 años de edad, con cáncer y neutropenia grave post-quimioterapia, que presentaban patología pulmonar aguda. El LBA se indicó en pacientes con: procesos pulmonares difusos, en procesos pulmonares
Hospital San Borja Arriarán Santiago (CLA, PS). Hospital Exequiel González Cortés, Santiago (MZ, CS). Hospital San Juan de Dios, Santiago (AMA, MV). Hospital Sótero Del Río, Santiago (AB, TV).

Hospital Luis Calvo Mackenna, Santiago (MES, MV) Hospital Roberto del Río, Santiago (ST, JT).

Los autores declaran no tener potenciales conflictos de interés. Auspicio: Fundación Nuestros Hijos.

Recibido: 27 de junio de 2011 Aceptado: 31 de marzo de 2012

Correspondencia a: Carmen Luz Avilés Lohmann claviles|@gmail.com 
localizados refractarios sin respuesta clínica luego de 72 horas de tratamiento antimicrobiano y en pacientes con procesos pulmonares que aparecieran después del séptimo día de evolución de neutropenia febril, de acuerdo a las indicaciones establecidas en el Consenso Nacional Manejo Racional del Paciente con Neutropenia Febril de $2005^{2}$.

Se obtuvo datos de edad, sexo, diagnóstico oncológico de base, síntomas clínicos y duración de éstos, tipo de imágenes pulmonares en la radiografía y en la TAC de tórax, diagnóstico final, descripción de las complicaciones asociadas al LBA y eventuales cambios de conducta terapéutica en relación a los hallazgos del LBA.

Se obtuvo consentimiento informado para la realización de cada procedimiento, de acuerdo a la normativa actual, de uno de los padres o tutor legal.

El LBA fue realizado en cada uno de los hospitales por médicos especialistas en patología broncopulmonar, empleando fibrobroncoscopio pediátrico flexible y usando técnica estándar ${ }^{4}$. El fibrobroncoscopio fue localizado en las zonas de interés según la información imagenológica o en el lóbulo medio, instilándose por el canal de trabajo tres alícuotas de $1 \mathrm{ml} / \mathrm{kg}$ de solución salina fisiológica $(\mathrm{NaCl}$ 9\%o) estéril. Inmediatamente después de cada instilación se aspiró por el mismo canal de trabajo a un tubo $\mathrm{T}$ de vidrio interpuesto, con la válvula de aspiración del equipo conectada a la red central. Usualmente se recuperan volúmenes equivalentes al $30-50 \%$ de lo instilado ${ }^{10,11}$.

El líquido recuperado se envió a estudio de laboratorio que incluye citología con citocentrifugado y teñido con May Grünwald-Giemsa, cultivo bacteriológico cuantitativo $^{2}$ en agar sangre, agar chocolate y Mac-Conkey. Se consideró cultivo positivo aquel cultivo cuantitativo con valor sobre $10^{4}$ unidades formadoras de colonias (ufc) $/ \mathrm{ml}^{12}$. Se efectuó estudio para hongos con tinción hematoxilina-eosina, Gomori Grocott y cultivo (agar Sabouraud), baciloscopia y cultivo para Mycobacterium tuberculosis (TBC), tinción Gomori Grocott para Pneumocystis jiroveci ${ }^{\mathrm{a}}$, cultivo acelerado en células humanas MRC-5 (shell vial ${ }^{\mathrm{b}}$ ) para citomegalovirus (CMV), reacción de polimerasa en cadena (RPC) para $M$. tubercuosis $^{\mathrm{c}}$, P. jiroveci ${ }^{\mathrm{d}}$, Chlamydia pneumoniae, Mycoplasma pneumoniae $\mathrm{y}^{\mathrm{e}}$ metapneumovirus . $^{\mathrm{b}}$

En forma complementaria se realizó inmunofluorescencia viral directa (IFD) de aspirado nasofaríngeo y galactomanano (GM) para Aspergillus en suero por un ELISA doble sandwich directo (Platelia Aspergillus ${ }^{\circledR}$; Bio Rad Marnes-La-Coquette France) en muestras seriadas de suero considerándose positivo dos muestras con un valor de corte $\mathrm{DO}>1^{13}$.

Se evaluaron los hallazgos microbiológicos según el tipo de infiltrado encontrado en la radiografía y/o TAC de tórax catalogados de infiltrados focales o intersticiales

Se analizó la utilidad clínica del LBA considerando los resultados microbiológicos, el rendimiento y la relación entre los resultados y los cambios de conducta terapéutica, reportándose las complicaciones relevantes asociadas al procedimiento.

\section{Resultados}

Se efectuaron 37 lavados en 35 pacientes oncológicos pediátricos, $60 \%$ de sexo masculino, con una mediana de 8 años (rango: 1-17 años) (Tabla 1). La mediana de duración de los síntomas previo a la realización del LBA fue de 7 días, (rango: 0-20 días). En la mayoría de los episodios, $35 / 37$, los pacientes estaban en tratamiento con antimicrobianos de amplio espectro, al menos por tres días antes del procedimiento y 22 de ellos con tratamiento antifúngico. Al momento del LBA, seis pacientes se encontraban en ventilación mecánica.

La patología de base correspondió a leucemia linfoblástica aguda (n: 10), leucemia mieloide aguda (n: 9), recaída de leucemia linfoblástica aguda (n: 6) y un paciente por cada uno de los siguientes diagnósticos: linfoma no Hodgkin, histiocitosis, leucemia mieloide crónica, mielodisplasia, sarcoma de Ewing, sarcoma de tejidos blandos, tumor de sistema nervioso central, hepatoblastoma, recaída de tumor sólido y tumor rabdoide intestinal.

Tabla 1. Características clínicas de 35 pacientes con

cáncer, neutropenia e imágenes pulmonares sometidos a lavado bronco-alveolar. PINDA 2005-2008

\begin{tabular}{|lll|}
\hline Características & & $\mathbf{n}(\%)$ \\
Sexo & Masculino & $21(60)$ \\
& Mediana & 8 años \\
Diagnóstico & Rango & 1 año a 17 años \\
& LLA & 10 \\
& LMA & 9 \\
& LLA en recaída & 6 \\
& Otros & 10 \\
\hline LLA: Leucemia linfoblástica aguda. LMA: Leucemia mieloide aguda.
\end{tabular}

\footnotetext{
a En laboratorios locales de cada uno de los centros hospitalarios.

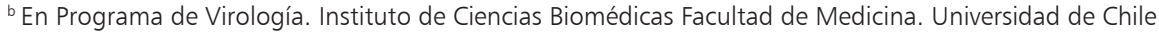

cEn laboratorio de Microbiología Hospital del Tórax.

d En Programa de Microbiología. Laboratorio de Infecciones Respiratorias. Instituto de Ciencias Biomédicas Facultad de Medicina. Universidad de Chile.

e Programa de Microbiología, Instituto de Ciencias Biomédicas, Facultad de Medicina, Universidad de Chile.
} 
En 28 de los 37 LBA no se realizaron todos los estu- insuficiente (Tabla 2). El estudio citológico y teñido con dios considerados en el protocolo, por diversos factores, May Grünwald-Giemsa no se realizó en la mayoría de los especialmente porque el volumen de la muestra era casos por lo cual estos datos no son analizados.

Tabla 2. Resultados microbiológicos en 35 pacientes pediátricos con cáncer, neutropenia e imágenes pulmonares sometidos a LBA. PINDA 2005-2008

\begin{tabular}{|c|c|c|c|c|c|c|c|c|c|c|c|}
\hline$\stackrel{n}{\text { LBA }}$ & Cultivo & $\begin{array}{l}\text { Shell vial } \\
\text { CMV }\end{array}$ & $\begin{array}{c}\text { RPC } \\
\text { Metapneu- } \\
\text { movirus }\end{array}$ & IFV & $\begin{array}{l}\text { BK, } \\
\text { Cultivo } \\
\text { TBC }\end{array}$ & $\begin{array}{l}\text { RPC } \\
\text { TBC }\end{array}$ & $\begin{array}{l}\text { Cultivo } \\
\text { hongos }\end{array}$ & GM & $\begin{array}{c}\text { RPC } \\
\text { P. jiroveci }\end{array}$ & $\begin{array}{c}\text { RPC } \\
\text { Chla- } \\
\text { mydia }\end{array}$ & $\begin{array}{c}\mathrm{RPC} \\
\text { Myco- } \\
\text { plasma }\end{array}$ \\
\hline 1 & Neg & $\mathrm{Nr}$ & $\mathrm{Nr}$ & Neg & Neg & $\mathrm{Nr}$ & Neg & Neg & Neg & Neg & Neg \\
\hline 2 & $P a 10^{4}, A b 10^{5}$ & Neg & Neg & Neg & $\mathrm{Nr}$ & Neg & Neg & $\mathrm{Nr}$ & Neg & $\mathrm{Nr}$ & $\mathrm{Nr}$ \\
\hline 3 & Neg & $\mathrm{Nr}$ & $\mathrm{Nr}$ & Neg & Neg & $\mathrm{Nr}$ & $\mathrm{Neg}$ & $\mathrm{Nr}$ & $\mathrm{Neg}$ & $\mathrm{Nr}$ & $\mathrm{Neg}$ \\
\hline 4 & S a $10^{3}$ & Neg & Neg & Neg & Neg & $\mathrm{Nr}$ & Neg & Neg & Neg & Neg & Neg \\
\hline 5 & Neg & Neg & Neg & $\mathrm{Nr}$ & $\mathrm{Nr}$ & $\mathrm{Nr}$ & Neg & Neg & Neg & Neg & Neg \\
\hline 6 & Neg & Neg & Neg & Neg & Neg & $\mathrm{Nr}$ & Neg & Neg & Neg & Neg & Neg \\
\hline 7 & Neg & Neg & $\mathrm{Nr}$ & Neg & $\mathrm{Nr}$ & Neg & Neg & $\mathrm{Nr}$ & Neg & $\mathrm{Nr}$ & $\mathrm{Nr}$ \\
\hline 8 & Neg & $\mathrm{Nr}$ & $\mathrm{Nr}$ & Neg & Neg & Neg & Neg & $\mathrm{Nr}$ & Neg & Neg & Neg \\
\hline 9 & Neg & Neg & Neg & Neg & Neg & $\mathrm{Nr}$ & Neg & Neg & Neg & Neg & Neg \\
\hline 10 & Neg & Neg & $\mathrm{Nr}$ & Neg & $\mathrm{Nr}$ & Neg & Neg & $\mathrm{Nr}$ & $\mathrm{Nr}$ & $\mathrm{Nr}$ & $\mathrm{Nr}$ \\
\hline 11 & Neg & Neg & Neg & Neg & Neg & Neg & Neg & $\mathrm{Nr}$ & Neg & Neg & Neg \\
\hline 12 & A. $/ 10^{4}$ & Neg & Neg & Neg & Neg cultivo Pos & Neg & Neg & Neg & Neg & Neg & Neg \\
\hline 13 & Neg & $\mathrm{Nr}$ & $\mathrm{Nr}$ & $\mathrm{Nr}$ & $\mathrm{Nr}$ & $\mathrm{Nr}$ & $\mathrm{Nr}$ & $\mathrm{Nr}$ & Neg & $\mathrm{Nr}$ & $\mathrm{Nr}$ \\
\hline 14 & P a no cuant. & Neg & Neg & $\mathrm{Nr}$ & $\mathrm{Nr}$ & $\mathrm{Nr}$ & Neg & $\mathrm{Nr}$ & $\mathrm{Nr}$ & $\mathrm{Nr}$ & Neg \\
\hline 15 & Pa $10^{6}$ & Neg & Neg & Neg & Neg & Neg & Neg & $\mathrm{Nr}$ & Neg & Neg & Pos \\
\hline 16 & Neg & Pos & $\mathrm{Nr}$ & $\mathrm{Nr}$ & Neg & Neg & Neg & $\mathrm{Nr}$ & Pos & Neg & Neg \\
\hline 17 & Neg & Neg & Neg & Neg & Neg & Neg & Neg & $\mathrm{Nr}$ & Neg & Neg & Neg \\
\hline 18 & Neg & Neg & Pos & Neg & Neg & Neg & Neg & Neg & Neg & Neg & Neg \\
\hline 19 & Neg & Neg & $\mathrm{Nr}$ & Neg & Neg & Neg & Neg & $\mathrm{Nr}$ & Neg & $\mathrm{Nr}$ & $\mathrm{Nr}$ \\
\hline 20 & Neg & Neg & Pos & VRS & Neg & $\mathrm{Nr}$ & Neg & $\mathrm{Nr}$ & Neg & Neg & Neg \\
\hline 21 & S. $v i r>10^{5}$ & Neg & Neg & Neg & Neg & Neg & Neg & Neg & Neg & Neg & Neg \\
\hline 22 & A / no cuant. & Neg & Neg & VRS & Neg & $\mathrm{Nr}$ & Neg & Neg & Neg & Neg & Neg \\
\hline 23 & Neg & $\mathrm{Nr}$ & Neg & Neg & Neg & Neg & Neg & Pos (2) & $\mathrm{Nr}$ & Neg & Neg \\
\hline 24 & S. vir $8 \times 10^{3}$ & $\mathrm{Nr}$ & $\mathrm{Nr}$ & Neg & Neg & Neg & Neg & Pos (2) & $\mathrm{Nr}$ & Neg & Neg \\
\hline 25 & Neg & Neg & Neg & Neg & Neg & Neg & Neg & Neg & Neg & Neg & Neg \\
\hline 26 & Neg & Neg & Neg & Neg & Neg & Neg & Neg & $\mathrm{Nr}$ & Neg & Neg & Neg \\
\hline 27 & Neg & Neg & Neg & Neg & Neg & Neg & Candida a. & Neg & Neg & Neg & Neg \\
\hline 28 & Neg & Neg & Neg & Neg & $\mathrm{Nr}$ & Neg & Neg & Neg & Neg & Neg & Neg \\
\hline 29 & Neg & Neg & $\mathrm{Nr}$ & $\mathrm{Nr}$ & Neg & Neg & Neg & $\mathrm{Nr}$ & Pos & $\mathrm{Nr}$ & $\mathrm{Nr}$ \\
\hline 30 & Neg & $\mathrm{Nr}$ & Neg & $\mathrm{Nr}$ & Neg & Neg & Neg & Pos (2) & Neg & Neg & Neg \\
\hline 31 & Neg & $\mathrm{Nr}$ & Neg & Neg & Neg & Neg & Aspergillus $n$. & $\operatorname{Pos}(2)$ & Neg & Neg & Neg \\
\hline 32 & Neg & $\mathrm{Nr}$ & Neg & $\mathrm{Nr}$ & Neg & $\mathrm{Nr}$ & Neg & $\mathrm{Nr}$ & Neg & Neg & Neg \\
\hline 33 & Neg & $\mathrm{Nr}$ & $\mathrm{Nr}$ & Neg & $\mathrm{Nr}$ & $\mathrm{Nr}$ & $\mathrm{Nr}$ & Neg & $\mathrm{Nr}$ & Neg & Neg \\
\hline 34 & Aspergillus $f$. & $\mathrm{Nr}$ & $\mathrm{Nr}$ & Neg & Neg & Neg & Aspergillus $f$. & Pos (2) & Neg & Neg & Neg \\
\hline 35 & Neg & $\mathrm{Nr}$ & $\mathrm{Nr}$ & $\mathrm{Nr}$ & Neg & Pos & Neg & $\operatorname{Pos}(2)$ & $\mathrm{Nr}$ & $\mathrm{Nr}$ & $\mathrm{Nr}$ \\
\hline 36 & Neg & Neg & $\mathrm{Nr}$ & Neg & Neg & Neg & Neg & Pos (2) & Neg & $\mathrm{Nr}$ & $\mathrm{Nr}$ \\
\hline 37 & Neg & Neg & $\mathrm{Nr}$ & $\mathrm{Nr}$ & Neg & $\mathrm{Nr}$ & Neg & Neg & Neg & $\mathrm{Nr}$ & $\mathrm{Nr}$ \\
\hline
\end{tabular}

CMV: citomegalovirus, RPC: reacción de polimerasa en cadena, BK: baciloscopia, TBC: tuberculosis, GM: galactomanano, Nr: no realizado, Neg: negativo, Pos: positivo, no cuant: no cuatitativo, Pa: Pseudomonas aeruginosa, Sa: Staphylococcus aureus, Al: Acinetobacter Iwoffii, S vir: Streptococcus grupo viridans, Aspergillus n: Aspergillus niger, Aspergillus f: Aspergillus fumigatus, Candida a: Candida albicans. 


\section{Hallazgos microbiológicos}

Hubo hallazgos microbiológicos significativos en 12/37 (32,4\%) de los LBA, identificándose 16 microorganismos (Tabla 3). En otros dos casos se encontraron cultivos positivos con recuentos no significativos para Staphylococcus aureus $\left(10^{3} \mathrm{ufc}\right)$ y Pseudomonas aeruginosa (cultivo no cuantitativo) con bacteriemia demostrada por hemocultivos en ambos casos. En otro episodio se obtuvo cultivo positivo con recuento bajo el punto de corte definido a $S$. grupo viridans $\left(10^{3} \mathrm{ufc}\right)$.

En ocho procedimientos se encontró un agente único y en cuatro una infección mixta.

\begin{tabular}{|c|c|c|c|c|}
\hline Paciente & Sexo & $\begin{array}{l}\text { Edad } \\
\text { años }\end{array}$ & $\begin{array}{l}\text { Diagnóstico } \\
\text { de base }\end{array}$ & Resultados \\
\hline 1 & $\mathrm{~F}$ & 8 & LLA & $P$ aeruginosa $1 \times 10^{4}$ ufc y $A$. baumannii $1 \times 10^{5}$ ufc \\
\hline 2 & $\mathrm{~F}$ & 14 & LMA & A. Iwoffii $8 \times 10^{4}$ ufc y $M$. tuberculosis \\
\hline 3 & M & 9 & LLA recaída & $P$. aeruginosa $>1 \times 10^{6}$ ufc y $M$. pneumoniae $(\mathrm{RPC}+)$ \\
\hline 4 & $\mathrm{~F}$ & 4 & LLA & Citomegalovirus y $P$. jiroveci (RPC + ) \\
\hline 5 & M & 6 & LLA & Metapneumovirus (RPC + ) \\
\hline 6 & $\mathrm{~F}$ & 4 & LLA & Metapneumovirus (RPC + ) \\
\hline 7 & $\mathrm{~F}$ & 5 & LLA & S. grupo viridans $>1 \times 10^{4}$ ufc \\
\hline 8 & M & 12 & LLA recaída & C. albicans \\
\hline 9 & $\mathrm{~F}$ & 1 & Histiocitosis & P. jiroveci (RPC +) \\
\hline 10 & M & 14 & LMA & Aspergillus niger \\
\hline 11 & M & 15 & LLA & Aspergillus fumigatus \\
\hline 12 & M & 12 & LMA & M. tuberculosis (RPC + ) \\
\hline
\end{tabular}

*Cultivo salvo que se indique otra técnica. LLA: Leucemia linfoblástica aguda. LMA: Leucemia mieloide aguda; ufc: Unidades formadoras de colonias. RPC: Reacción de polimerasa en cadena.

Tabla 4. Lavado bronco-alveolar BA en pacientes pediátricos con cáncer, neutropenia e imágenes pulmonares: correlación entre tipo de infiltrado radiológico pulmonar y patógenos identificados

\section{Tipos de infiltrado}

Infiltrado focal (n: 19)

Infiltrado intersticial (n: 15)

\section{Hallazgos microbiológicos}

A. baumannii y $P$. aeruginosa

$P$. aeruginosa y $M$. pneumoniae

A. Iwoffii y M. tuberculosis

S. grupo viridans

Aspergillus fumigatus

Aspergillus niger

M. tuberculosis (RPC)

\section{P. jiroveci}

P. jiroveci y citomegalovirus

Metapneumovirus

C. albicans

Infiltrado mixto (n: 2)

VRS y metapneumovirus
En detalle:

- Bacterias: en 4 de 37 procedimientos (LBA) se aislaron 5 bacterias en cultivos con recuentos significativos: $P$. aeruginosa (n: 2), A. baumannii (n: 1), A. lwoffii (n: 1) y Streptococcus grupo viridans (n: 1).

Mycoplasma pneumoniae se encontró en 1/27 LBA por técnica de RPC. Mycobacterium tuberculosis se aisló en cultivo en 1/29 LBA y hubo una RPC para M. tuberculosis positiva en $1 / 23$, con cultivo de Koch negativo.

La pesquisa de Chlamydia pneumoniae por RPC fue negativa en 26 LBA.

- Virus: Se efectuó estudio viral para CMV en 25 LBA encontrándose un shell vial positivo; en la misma muestra se identificó además $P$. jiroveci por RPC.

La RPC para metapneumovirus fue positiva en $2 / 22$ LBA.

La IFD por aspirado nasofaríngeo fue positiva en $2 / 28$ para VRS, en un caso concomitante con RPC para metapneumovirus.

- Hongos: en 3/37 LBA se aisló un hongo: Aspergillus fumigatus (n: 1), Aspergillus niger (n: 1) y Candida albicans (n: 1), respectivamente.

Se detectó además $P$. jiroveci por RPC en 2/30 LBA. El estudio de galactomanano fue positivo en $7 / 21$ episodios, con índice DO entre 1,4 y 14,7 con una mediana 3,5, en dos casos se asoció con aislamiento de A. fumigatus (n: 1) y A. niger (n: 1), respectivamente. Los otros cinco casos de GM positivo, sin cultivo concomitante, tenían un cuadro clínico compatible con infección fúngica y valores igualmente elevados en las dos determinaciones y fueron diagnosticados como tales.

\section{Tipo de imagen pulmonar y hallazgos etiológicos} (Tabla 4)

Infiltrados focales: En 19/37 casos se consignaron infiltrados focales. En 7 se encontró un microorganismo a través del LBA, en 5 casos se demostró una bacteria y en dos una especie fúngica.

En otros 5 casos se estableció la etiología del episodio por métodos como GM (n: 3) o hemocultivos (n: 2).

En síntesis, en 7/19 episodios con imágenes focales se demostró una infección bacteriana y en 5/19 micótica.

Infiltrados intersticiales difusos En 15/37 casos se encontró infiltrados intersticiales. En 4 se detectó un microorganismo: metapneumovirus (n: 1), hongos (n: 2, C. albicans y $P$. jiroveci) e infección mixta (n: $1, P$. jiroveci y citomegalovirus). En los 11 restantes el resultado fue negativo.

En dos episodios se informaron lesiones mixtas (con- 
solidantes e intersticiales), uno correspondió a infección por metapneumovirus y VRS y el otro no tuvo etiología.

En un caso el paciente presentaba masa mediastínica sin infiltrados pulmonares y no se llegó a diagnóstico por LBA.

En 6/37 episodios el resultado del LBA permitió fundamentar un cambio de conducta terapéutica con respecto al esquema inicial, en dos casos se suspendió el tratamiento antimicrobiano, en uno se inicia ganciclovir, en otro se agrega cotrimoxazol y en dos voriconazol.

Las técnicas de biología molecular permitieron identificar 6 de los $16(37,5 \%)$ microorganismos detectados: $P$. jiroveci (2), metapneumovirus (2), TBC (1) Mycoplasma pneumoniae (1).

\section{Seguridad del procedimiento}

No hubo complicaciones de importancia relacionadas al procedimiento en 32/37 LBA. En un caso se presentó hemorragia pulmonar sin que pudiese atribuirse al procedimiento y en cuatro los datos clínicos fueron insuficientes para evaluar la seguridad del LBA.

El LBA efectuado a los seis pacientes en ventilación mecánica fue bien tolerado.

\section{Discusión}

La FBC con LBA en pacientes pediátricos con cáncer y neutropenia grave tiene un lugar importante en la identificación de la etiología de infiltrados pulmonares, dado que en estos pacientes es fundamental un diagnóstico rápido y seguro para efectuar tratamientos efectivos, considerando las múltiples posibilidades etiológicas.

El rendimiento del LBA por FBC en pacientes pediátricos con cáncer cursando una infección respiratoria aguda baja en distintos reportes oscila entre 30 y 53\% ${ }^{5,7,8,14}$. En el presente estudio se obtuvo un rendimiento de $32,4 \%$ en $37 \mathrm{LBA}$, resultados comparables a lo señalado en la literatura médica. El rendimiento del LBA podría ser mejorado corrigiendo factores tales como la oportunidad en la realización del procedimiento, que en nuestro estudio se hizo hasta 20 días después del inicio de la infección respiratoria baja, con pacientes recibiendo tratamientos antibióticos y antifúngicos empíricos. Lo recomendable es hacer este procedimiento lo más precozmente posible ${ }^{15}$. Otro elemento a considerar que afecta el rendimiento es el volumen de la muestra necesario para realizar el protocolo de estudio completo; es difícil de alcanzar el volumen requerido para completar todos los análisis propuestos por condiciones propias de la técnica.

Los hallazgos etiológicos múltiples, frecuentes en pacientes inmunocomprometidos pueden ser discutibles, no siendo fácil interpretar si se trata o no de una contaminación. Los episodios con cultivos positivos sin recuento cuantitativo o con recuentos menores al punto de corte definido $\left(10^{4} \mathrm{ufc} / \mathrm{ml}\right)$, no fueron incluidos en los resultados de esta serie. Existe controversia al respecto ya que, aunque su inclusión aumentaría la sensibilidad del LBA, la interpretación de los resultados requiere un cuidadoso análisis para no disminuir la especificidad ${ }^{16,17}$. En pacientes inmunocomprometidos, los cultivos cuantitativos no debieran ser el único criterio para definir el significado de un cultivo positivo sino que deben considerarse además criterios clínicos, las características de las imágenes y los resultados de los análisis de laboratorio ${ }^{18,19}$.

Se reportaron cambios en la conducta terapéutica en relación a resultados del LBA en seis de 37 pacientes $(16,2 \%)$ en quienes se iniciaron nuevas terapias o se suspendieron tratamientos instaurados empíricamente. La obtención de resultados negativos también puede ser de utilidad permitiendo suspender tratamientos empíricos injustificados, ya que valida la ausencia de patógenos bacterianos, virales o micóticos ${ }^{7}$.

El uso temprano y sistemático del LBA por FBC con técnicas microbiológicas cuantitativas y de biología molecular, mejora los resultados etiológicos permitiendo tratamientos más oportunos y específicos ${ }^{20,21}$. En nuestra serie a pesar del retraso en su realización, en 32,4\% de los pacientes se identificó etiología infecciosa por LBA.

El LBA en pacientes con hallazgos radiológicos focales tuvo mejor rendimiento que en los casos con infiltrados intersticiales, la literatura científica reporta resultados diversos, en unos casos con mayor utilidad en hallazgos focales $^{7} \mathrm{y}$ en otras ocasiones en imágenes multifocales ${ }^{22}$.

El procedimiento es seguro en pacientes oncológicos incluso en condiciones de insuficiencia respiratoria; $\sin$ embargo, en este estudio no se realizó un análisis acabado de las complicaciones menores que pudieran haberse presentado, como hipoxemia leve o fiebre ${ }^{8}$.

En suma, el LBA tiene un importante papel en la evaluación de los infiltrados pulmonares en pacientes pediátricos con cáncer y neutropenia febril, en quienes un diagnóstico rápido y seguro es crucial en la sobrevida. Sugerimos que el LBA por FBC sea considerado como una herramienta diagnóstica inicial en estos pacientes críticamente enfermos. Se destaca la importancia de realizar un estudio protocolizado que permita efectuar en forma simultánea un amplio estudio etiológico, tanto con cultivos cuantitativos como con técnicas de biología molecular para optimizar los resultados.

\section{Resumen}

Las infecciones pulmonares en niños con cáncer son una complicación grave. El lavado broncoalveolar (LBA) es un procedimiento efectivo para llegar a un diagnóstico etiológico. Se analizaron los resultados de LBA realizados entre noviembre de 2005 y octubre de 2008 , en niños con 
cáncer y neutropenia grave e infiltrados pulmonares para conocer su rendimiento, utilidad clínica y seguridad. Se evaluaron 37 LBA en 35 pacientes. En 19/37 casos los infiltrados radiológicos fueron focales, en 8 se encontró etiología por LBA. En 15/37 casos las imágenes fueron intersticiales encontrándose etiología en 4 , resultando un rendimiento microbiológico superior en las lesiones focales. Las muestras del LBA fueron positivas en 32,4\% de los episodios (12/37). Se detectaron 16 microorganismos: 8 bacterias, a saber Mycobacterium tuberculosis (n: 2), Pseudomonas aeruginosa (n: 2), Acinetobacter baumannii (n: 1), A. lwoffii (n: 1), Streptococcus grupo viridans (n: 1) y Mycoplasma pneumoniae (n: 1); 3 virus: metapneumovirus (n: 2) y citomegalovirus (n: 1); 5 hongos: Pneumocystis jiroveci (n: 2), Aspergillus fumigatus (n: 1), Aspergillus niger (n: 1) y Candida albicans (n: 1). Se reportaron cambios en la conducta terapéutica en 6 de 37 pacientes $(16,2 \%)$ con inicio de nuevas terapias o suspensión de tratamientos empíricos. El LBA tuvo un adecuado rendimiento, sin complicaciones importantes por lo que debe ser considerado precozmente y realizado con un estudio protocolizado.

\section{Referencias bibliográficas}

1.- Maschmeyer G, Beinert T, Buchheidt D. Diagnosis and antimicrobial therapy of lung infiltrates in febrile neutropenic patients: Guidelines of the Infectious Diseases Working Party of the German Society of Haematology and Oncology. Eur J Cancer 2009; 45: 2462-72.

2.- Santolaya M E, Rabagliati R, Bidart T. Consenso Nacional Manejo Racional del Paciente con Neutropenia Febril . Rev Chilena Infectol 2005; 22 (Supl 2): S79-S113.

3.- De Blic J, Midulla F, Barbato A. Bronchoalveolar lavage in children. ERS Task Force on Bronchoalveolar Lavage in Children. European Respiratory Society. Eur Respir J 2000; 15: 217-31.

4.- Prado F, Boza M L, Badilla J. Fibrobroncoscopia en pediatría: utilidad diagnóstica y terapéutica. Rev Chil Pediatr 1998; 69 (5): 207-14.

5.- Vega L, Holmgren N. Utility of bronchoalveolar lavage in immunocompromised children: diagnostic yield and complications. Arch Bronconeumol 2004; 40: 570-4.

6.- Sánchez I, Pesce C, Navarro H. Experiencia en 10 años de aplicación de fibrobroncoscopia en pacientes pediátricos. Rev Med Chile 2003; 131 (11): 1266-72.

7.- Park J, Fogarty S, and Brogan T. Clinical utility of bronchoalveolar lavage in pediatric cancer patients. Med Ped Oncol 2002; 39: 175-80.

8.- Efrati O, Gonik U, Bielorai B. A fiberoptic bronchoscopy and bronchoalveolar lavage for the evaluation of pulmonary disease in children with primary immunodeficiency and cancer. Pediatr Blood Cancer 2007; 48: 324-9.

9.- Efrati O, Sadeh-Gornik U, Modan-Moses D. Flexible bronchoscopy and bronchoalveolar lavage in pediatric patients with lung disease. Pediatr Crit Care Med 2009 Jan; 10: 80-4.

10.- Balfour-Lynn I, Spencer H. Bronchoscopy-how and when? Pediatric Respir Rev 2002; 3 : 255-64.

11.- Nicolai T. Pediatric bronchocopy. Pediatr Pulmonol 2001; 31: 150-64.

12.- Fica A, Cifuentes M, Hervé B. Actualización del Consenso Neumonía Asociada a Ventilación Mecánica. Primera parte. Aspectos diagnósticos. Rev Chil Infect 2011; 28 (2): 130-51.

13.- Maertens J, Theunissen K, Verbeken E. Prospective clinical evaluation of lower cut-offs for galactomannan detection in adult neutropenic cancer patients and haematological stem cell transplant recipients. Br J Haematol 2004; 126: 852-60.

14.- Zamorano A, Núñez A, Salinas M. Experiencia clínica de la utilidad del lavado broncoalveolar en pediatría. Rev Chil Pediatr 2002; 73: 576-82. 15.- Rosenthal M. Bronchoscopy and infection.
Paediatr Respir Rev 2003; 4: 143-6.

16.- Zedtwitz K, Schenk P, Apfalter P. Ventilatorassociated pneumonia: increased bacterial count in bronchoalveolar lavage by using urea as an endogenous marker of dilution. Crit Care Med 2005; 33: 756-9.

17.- Shigeki F, Yu V. Quantitative cultures for diagnosing ventilator-associated pneumonia: A critique. Clin Infect Dis 2006; 43: S106-S113.

18.- Bochennek K, Abolmaali N, Wittkindt B. Diagnostic approaches for immunocompromised paediatric patients with pulmonary infiltrates. Clin Microbiol Infect 2006; 12: 199-201.

19.- Lisboa T, Rello J. Diagnosis of ventilatorassociated pneumonia: is there a gold standard and a simple approach? Curr Opin Infect Dis 2008; 21: 174-8.

20.- Hohenthal U, Itala M, Salonen J. Bronchoalveolar lavage in immunocompromised patients with haematological malignancy. Value of new microbiological methods. Eur J Haematol 2005; 74: 203-11.

21.- Kuehnhardt D, Hannemann M. Therapeutic implication of BAL in patients with neutropenia. Ann Hematol 2009; 88: 1249-56.

22.- Peikert T, Rana S, Edell E. Safety, diagnostic yield and therapeutic implications of flexible bronchoscopy in patients with febrile neutropenia and pulmonary infiltrates. Mayo Clin Proc 2005; 80 (11): 1414-20. 